\title{
Geometrical Effects in Mechanical Characterizing of Microneedle for Biomedical Applications
}

\author{
Priyanka Aggarwal $^{*}$, C. R. Johnston ${ }^{* *}$ \\ *Department of Electrical and Computer Engineering, aggarwa@enel.ucalgary.ca \\ ${ }^{* *}$ Department of Mechanical Engineering, johnston@enme.ucalgary.ca \\ University of Calgary \\ 2500, University Drive, Calgary, Alberta, Canada, T2N1N4
}

\begin{abstract}
This paper discusses the design and implementation of MEMS microneedle with emphasis on integrated functionality for sensing the forces. The idea is based on the requirements of strength, robustness, and minimal insertion pain and tissue damage in patients The constraints of minimal microneedle dimension, force withstanding capabilities and their relations are the main basis for final design dimensions. Therefore, the design gives the efficient dimensions of the microneedle, while considering the influence of various mechanical forces and skin resistive forces. The design is extended by giving complete fabrication steps of the proposed microneedle design. Three piezoelectric sensors are fabricated on the microneedle, which reliable skin resistance measurement for different insertion depth. Performance analysis at the end proves the validity of the proposed design approach for different geometries and sizes.
\end{abstract}

Keywords: microneedles, mechanical forces, finite element modeling, skin resistance, piezoelectric sensing

\section{INTRODUCTION}

MicroElectroMechanical Systems (MEMS) is the integration of mechanical elements, sensors, actuators and electronics on a common silicon substrate through microfabrication technology [1]. The primary motivation for the development of microneedle is the need for needles that are economical to fabricate and which are small enough to reduce insertion pain and tissue trauma.

Today, the smallest conventional needle commercially available have a $305 \mu \mathrm{m}[2]$ outer diameter with a wall thickness of $76 \mu \mathrm{m}$. Since traditional machining methods limit the diameter of the manufactured needles to $300 \mu \mathrm{m}$, reductions in the size of the needles to ease the invasive effects of the needle are difficult to achieve with these methods. Further, it is extremely difficult to design and fabricate complex structures within a conventional needle.

On the other hand, microneedles are defined lithographically, so they can be designed for smaller size and geometry. Microneedles are extremely sharp, with submicron tip radii, as the fluid outlet is on the side of the needle. In addition, arrays of micro-needles with multiple outlet ports allow the injection of different fluids from the same device. Complex needle geometries such as 90-degree bends can be easily incorporated in microneedles [2].

In this work, performance analysis is made to validate the proposed design approach for different microneedle geometries and sizes. In addition, piezoelectric sensors are used to give reliable skin resistance measurement with the distance transversed into human skin.

This paper is organized into five Sections. Section 1 is the introduction. The various design issues related to selection of microneedle dimensions is covered in Section 2. The simulation results indicating the variation of forces are shown in Section 3. A brief overview of the fabrication steps are given in Section 4 and the paper is concluded in Section 5.

\section{MICRONEEDLE DESIGN/FAILURE}

Various factors are taken into account during the design of microneedles. Microneedles are far more reliable and efficient for transdermal drug delivery because of the small size. Also, microneedles may be used for obtaining precise blood samples, which are very helpful in the diagnosis of diseases. This paper presents the optimal design for the MEMS based microneedles. It takes into consideration the influence of mechanical forces (as compressive force, buckling force, free bending force, constrained bending and shear force) acting on the needle during insertion into human skin as well as skin resistive force. In addition, a comparison is drawn between several geometries and sizes of the microneedle.

The theoretical pressure required to pierce human skin has been given [3] as $3.183 * 10^{6} \mathrm{~Pa}\left(P_{\text {Piercing }}\right)$. This pressure is a major factor in determining the overall geometry of the MEMS microneedle. This pressure decreases drastically once the skin is punctured. The length of our MEMS based in-plane microneedle have been set at $600 \mu \mathrm{m}$ so as to withdraw blood samples from the capillaries in the dermis layer, which occurs at a distance of 500 to $2000 \mu \mathrm{m}$ below the skin surface. These microneedles are long enough to withdraw sufficient bodily fluids from the dermis layer and yet small enough not to puncture pain causing nerves found in deeper tissues. Piezoelectric sensors are configured over the microneedle to detect the deforming forces acting on the microneedle as it penetrates through human body. 


\subsection{Modes of Loading}

Analytically, microneedle dimensions can be calculated from the theoretical analysis for the compressive force, buckling, free bending, constrained bending and shear force, for a hollow beam with a fixed end. The tip force (exerted by the human skin) has to be smaller than any of these breaking forces. We have considered all of the abovementioned forces and found that the forces with the most impact on the determination of the dimensions of the structure are the buckling and the bending forces as defined below.

The maximum Euler's buckling [2,4] force that a microneedle can withstand without breaking is given by the following equation:

$$
F_{\text {MaxBuck }}=\frac{C \pi^{2} E I}{L^{2}}
$$

where $\mathrm{E}=169 \mathrm{GPa}$ represents the Young's Modulus of silicon, I $\left(\mathrm{m}^{4}\right)$ is the Moment of Inertia for different geometries as stated in Table 1, and L (m) is the length of the microneedle.

\begin{tabular}{|c|c|}
\hline Type of Microneedle & Moment of Inertia \\
\hline Circular Microneedle $^{1}$ & $I=\frac{\pi D^{4}-\pi d^{4}}{64}$ \\
\hline Rectangular Microneedle $^{2}$ & $I=\frac{B H^{3}-b h^{3}}{12}$ \\
\hline Square Microneedle $^{3}$ & $I=\frac{H^{4}-h^{4}}{12}$ \\
\hline
\end{tabular}

Table 1: Moment of Inertia for Type of Microneedle

In the computation, the base of the microneedle is modeled as a fixed joint giving end condition of $\mathrm{C}$ of 0.25 .

The maximum free bending force that the microneedle can withstand is given by equation $[2,4]$.

$$
F_{\text {MaxFreeBend }}=\frac{\sigma_{y} I}{c L}
$$

where $\mathrm{c}(\mathrm{m})$ is the distance of the neutral axis to the outermost edge of the microneedle. For a circular microneedle, $\mathrm{c}$ is $\mathrm{D} / 2$ where $\mathrm{D}$ is the outer diameter while for rectangular microneedle $\mathrm{c}$ is $\mathrm{H} / 2, \mathrm{H}$ being the outer height. For square, $\mathrm{c}$ is $\mathrm{H} / 2$ where $\mathrm{H}$ is the outer dimension. Also, $\sigma_{\mathrm{y}}$ is the yield strength of the microneedle made of silicon i.e $7 \mathrm{GPa}$.

The resistance offered by skin before skin being punctured is given by the following equation:

\footnotetext{
${ }^{1} \mathrm{D}$ is the outer diameter, $\mathrm{d}$ is inner diameter of microchannel running along length of microneedle

${ }^{2} \mathrm{~B}, \mathrm{H}$ are the outer width and height while $\mathrm{b}, \mathrm{h}$ are the inner width and height of the microchannel in the microneedle

${ }^{3} \mathrm{H}$ is the outer dimension while $\mathrm{h}$ is the inner dimension of the microchannel
}

$$
F_{\text {Skin }}=P_{\text {Piercing }} A
$$

where $\mathrm{A}$ is the cross sectional area of the microneedle. This force falls drastically once the skin is punctured $[5,6]$. After the skin is pierced, the only force that acts on the microneedle is the frictional force due to tissue clamping the needle. This post-puncturing reduces the pressure

$P_{\text {Post-Punctured }}$ to $1.6 \mathrm{MPa}[5,6]$ from the piercing pressure of $3.18 \mathrm{MPa}$.

\subsection{Piezoelectric Sensing}

High precision in the manual control of needles and biopsy probes in medical treatment requires high level of skill and dexterity. In anaesthesia, force sensation is an important feedback mechanism [5] and the practitioner needs to refresh or develop skills to improve the interpretation of the needles progress towards the target site. To have an idea of the force resisting progress of the microneedle into human skin, piezoelectric sensors have been included on the microneedle design. The progress of the microneedle in the body can be judged from the proportion of the microneedle remaining outside and force experienced during injection.

We know that, when a piezoelectric material is stressed mechanically by a force or moment, an electrical charge/voltage is generated. This is the basic sensing principle used in this design.

By expressing charges in terms of voltages, we get

$$
V_{12}=\frac{d_{33} F_{3} l_{1}}{\varepsilon \varepsilon_{o} A_{12}}
$$

where $\varepsilon$ is the permittivity of air, $\varepsilon_{0}$ is the relative permittivity of AlN and A is the area of the xy plane of the microneedle.

Here in this design, the force is being applied along $\mathrm{z}$ axis and the charge is measured along the $\mathrm{z}$ direction. Hence $d_{33}$ coefficient is being used.

The sensor design consists of three layers. A single, unimorph $1 \mu \mathrm{m}$ AlN layer is sandwiched between two electrodes layers each $0.8 \mu \mathrm{m}$ thick. The top layer consists of three strips of chromium electrode and the bottom layer has a single common aluminum electrode covering the entire area occupied by the top three electrode strips, which has been distributed along the top surface area of the microneedle. The area of intersection between the top and bottom electrodes is where the piezosensitive material is located. The AlN layer consists of three strips of $20 \mu \mathrm{m}$ width at interval of $300 \mu \mathrm{m}$. Three output voltages as given in Table 2 are obtained from this configuration of the sensing material. Here we are taking the length as $600 \mu \mathrm{m}$, cross sectional area of the microneedle to be $9575 \mu \mathrm{m}^{2}$. Also to calculate voltage for sensor $1, \mathrm{P}_{\text {Piercing }}$ is used while to calculate voltage for sensor 2 and sensor $3, P_{\text {Post-Punctured }}$ is being used. Fig 1 shows the variation of the three sensor voltages with the length of the microneedle. 


\begin{tabular}{|c|c|c|c|}
\hline Sensor & $\begin{array}{c}\text { Sensing } \\
\text { area }\left(\mu \mathrm{m}^{2}\right)\end{array}$ & $\begin{array}{l}\text { Position of } \\
\text { sensor }(\mu \mathrm{m})\end{array}$ & Voltage (V) \\
\hline Sensor 1 & 14500 & 1 & 0.1109 \\
\hline Sensor 2 & 8500 & 300 & 0.0952 \\
\hline Sensor 3 & 2900 & 600 & 0.2789 \\
\hline
\end{tabular}

Table 2: Voltages Obtained at Various Depths

As the length of the microneedle increases, the voltages decrease. Voltage V1 remains same as it is independent of length of the microneedle.

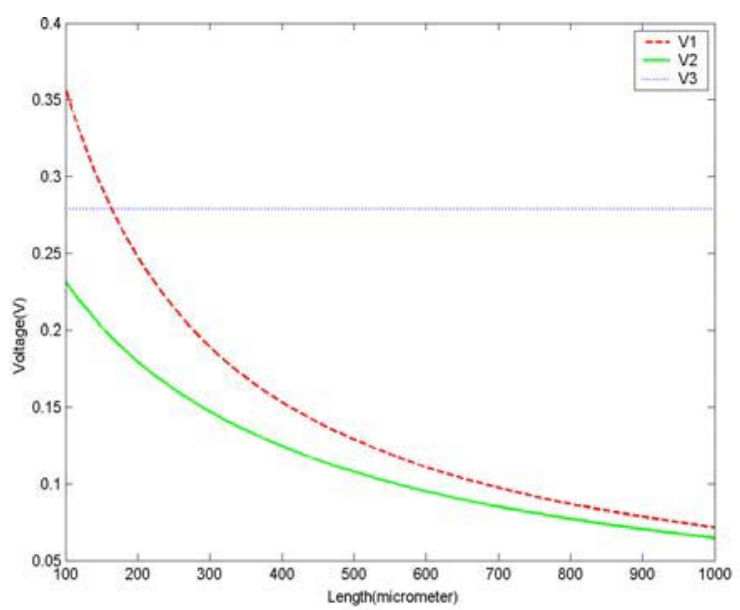

Figure 1: Variation of voltages

\section{SIMULATION RESULTS}

A cut-away view (showing the embedded channel) of a microneedle with a channel running along its length is shown in Fig 2. The dimensions of the microneedle are a width of $90 \mu \mathrm{m}$, height of $120 \mu \mathrm{m}$, and length of $600 \mu \mathrm{m}$, while the channel has a width and height of $35 \mu \mathrm{m}$. On substituting these values into the above mentioned formulas for rectangular microneedle, we get the maximum bending force $2.49 \mathrm{~N}$; buckling force of $14.851 \mathrm{~N}$ while the skin

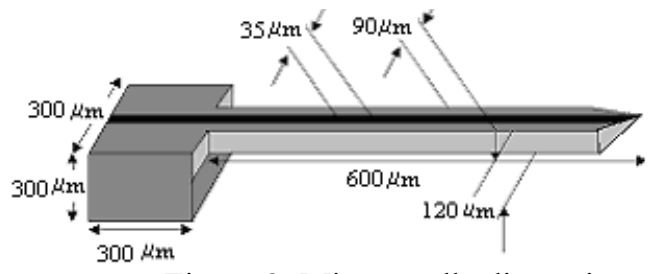

Figure 2: Microneedle dimensions

resistive force is $0.0305 \mathrm{~N}$, making the design sufficiently strong.

The same results are confirmed using ANSYS. These stresses are obtained when a bending force of $2.1 \mathrm{~N}$ and buckling force of $11.135 \mathrm{~N}$ is being applied on rectangular, square and circular microneedle along the $\mathrm{Y}$ axis, taking the yield strength of silicon to be $7 \mathrm{GPa}$ and young's modulus of $169 \mathrm{GPa}$. In Fig 3, the obtained yield stresses are plotted along the $\mathrm{Y}$ axis, keeping $\mathrm{X}$ coordinate at 0 and $\mathrm{Z}$ coordinate at $52 \mu \mathrm{m}$ (for Square microneedle), $45 \mu \mathrm{m}$ (for Rectangular microneedle) and $65 \mu \mathrm{m}$ (for Circular microneedle). These values are obtained on application of bending force of $2.1 \mathrm{~N}$ on the microneedles when the cross sectional area of the microneedles are kept same.

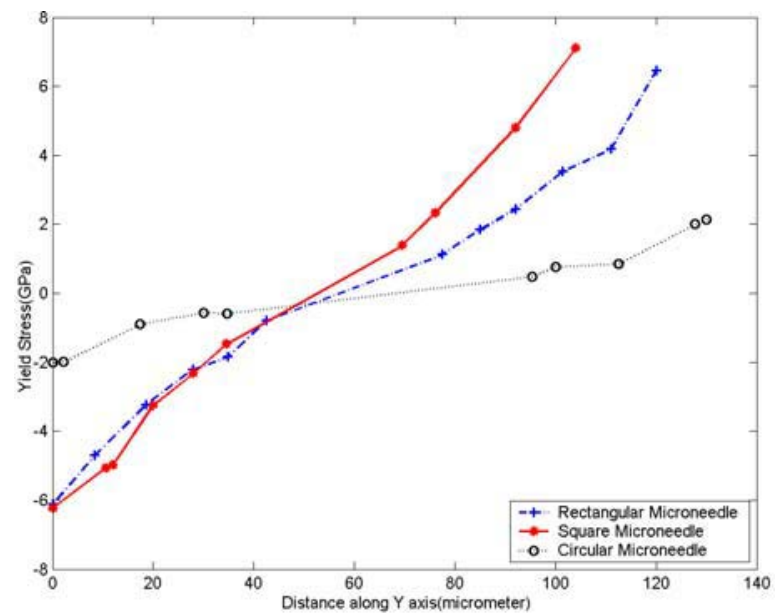

Figure 3: Yield Stresses for different shapes of microneedle

As can be seen in Fig 3, the values of stresses obtained for circular microneedle are less than those obtained for rectangular and square microneedles, indicating that circular microneedles can withstand more forces than rectangular or square microneedles.

In Fig 4, the Young's modulus of different shapes of the microneedle is plotted for varying distance along the $\mathrm{Y}$ axis, keeping $\mathrm{X}$ coordinate at 0 and $\mathrm{Z}$ coordinate at $52 \mu \mathrm{m}$ (for Square microneedle), $45 \mu \mathrm{m}$ (for Rectangular microneedle) and $65 \mu \mathrm{m}$ (for Circular microneedle). From Fig 4, it is seen that the Young's modulus for the circular microneedle is the highest among the three. This shows that circular microneedle has more strength when cross sectional area of the microneedles is kept same.

Although it is established that the circular microneedles can withstand more forces than either rectangular or square microneedle, we are fabricating rectangular microneedles, the next preferred shape because of the fabrication limitations.

\section{FABRICATION}

This section covers the steps necessary to fabricate the microneedle in order to verify the theoretical performance shown above. In the first step of fabrication, a $<100>$ silicon wafer is taken and cleaned with piranha. A layer of silicon dioxide of thickness $7500{ }^{\circ} \mathrm{A} \mathrm{[7]}$ is thermally grown at $900{ }^{\circ} \mathrm{C}$ for $4 \mathrm{~h}$ over the wafer. Then, the wafer is cleaned in a solution of $\mathrm{H}_{2} \mathrm{SO}_{4} / \mathrm{H}_{2} \mathrm{O}_{2}$ and $\mathrm{NH}_{4} \mathrm{OH} / \mathrm{H}_{2} \mathrm{O}_{2}$ and dried in an oven at $150^{\circ} \mathrm{C}$ for $10 \mathrm{~min}$. The oxide serves as an insulating layer. A layer of photosensitive material is spun onto the thermally deposited $\mathrm{Si}_{2} \mathrm{O}$ layer. 
A standard lithographic mask bearing the appropriate pattern to form the microchannel running along the length of the microneedle is positioned on top of the photoresist

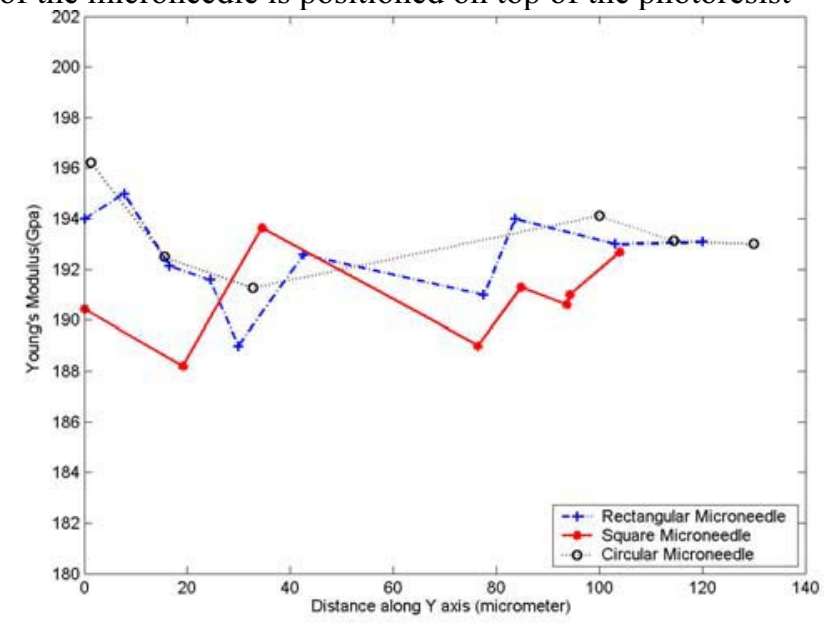

Figure 4: Young's Modulus for different shapes

layer. The wafer and photo-resist were then exposed to ultraviolet (UV) layer through the mask by means of optical mask aligner. The exposed photo-resist was removed by soaking the wafers in a liquid developer leaving the desired pattern of photo-resist. Now etching of the patterned oxide by DRIE [7] was done. This process forms the channel with the desired width $35 \mu \mathrm{m}$ and height $35 \mu \mathrm{m}$ in the bottom silicon wafer. The residual silicon dioxide is removed, followed by the necessary cleaning of the wafers before actual bonding takes place. The resulting wafer is fusion bonded with another silicon wafer [7]. The steps for the process include surface preparation, contact and finally annealing at $800{ }^{\circ} \mathrm{C}$. The $\mathrm{Si}$ fusion bonding [7] is based on tendency of smooth surfaces to adhere. It utilizes thermal cycling after contacting to increase bond strength. Once the two wafers are bonded, the top wafer is subsequently thinned down, to achieve the desired top wafer thickness of $40 \mu \mathrm{m}$.

The sensor structure consists of three layers. The fabrication starts with sputtering of aluminum electrode over the entire bonded wafer surface to achieve Al layer thickness of $8000{ }^{\circ} \mathrm{A}$. This forms the common bottom electrode layer for piezoelectric sensing of the forces as mentioned before. Magnetron sputtering of $\mathrm{Al}$ gives a deposition rate of $1 \mu \mathrm{m} / \mathrm{min}$ using the following specifications: pressure of $3 \mathrm{mT}$, power $9.5 \mathrm{KW}, \mathrm{Al}$ target and using argon at $30 \mathrm{sccms}$. Next, $1 \mu \mathrm{m}$ thick layer of AlN is deposited by magnetron sputtering. This layer is then patterned and etched using 1(conc.) HF acid: 1 water because this mixture etches the underlying $\mathrm{Al}$ at negligible rate as compared to AlN layer. Approximately $8000{ }^{\circ} \mathrm{A}$ thick chromium layer is deposited onto the AlN layer using DC sputtering to form the top electrode for the sensor. The sputtering parameters are: base pressure $1.2 \times 10^{-6} \mathrm{Torr}, \mathrm{Ar}$ [7] pressure $5 \mathrm{~m}$ Torr, substrate temperature $300{ }^{\circ} \mathrm{C}$ and sputtering power $400 \mathrm{~W}$. This layer is patterned and etched to form the top three electrodes using $(1: 3[50 \mathrm{~g} \mathrm{NaOH}+$ $\left.\left.100 \mathrm{ml} \mathrm{H}_{2} \mathrm{O}\right]:\left[30 \mathrm{~g} \mathrm{~K}_{3} \mathrm{Fe}(\mathrm{CN})_{6}+100 \mathrm{ml} \mathrm{H}_{2} \mathrm{O}\right]\right)$. This particular etchant is used in order to prevent etching of bottom AlN or Al layers. Now etching of unprotected bottom AlN and Al layers are carried out to form the desired shape. DRIE of the patterned silicon wafer is carried out using $\mathrm{C}_{4} \mathrm{~F}_{8}, \mathrm{SF}_{6}$, and $\mathrm{Ar}$ at etch rate of 2 $\mu \mathrm{m} / \mathrm{min}$, all the way down the wafer thickness to achieve vertical side walls. Finally, patterned backside etching. of the microneedle is carried out, to achieve the desired thickness of $120 \mathrm{um}$ for the length of $600 \mathrm{um}$.

\section{CONCLUSION}

The design/dimension criteria of in-plane micro-needles have been discussed in this paper. It is established that the circular microneedle can withstand more amount of force than either rectangular or square microneedles. The importance of the skin resistance on the dimensions (length, cross sectional area) of the microneedle is a novel approach not generally considered in microneedles design. Integrated sensor design for sensing the skin resistance at different depths inside the tissue has been presented.

\section{ACKNOWLEDGMENT}

The authors would like to thank K.V.I.S Kaler, Department of Electrical Engineering at University of Calgary and Natural Sciences and Engineering Research Council of Canada (NSERC) for their valuable guidance and support.

\section{REFERENCES}

[1] William S Trimmer, "Micromechanics and MEMS: Classic and Seminal Papers to 1990", IEEE press, 1997

[2] J.D.Zahn, N.H.Talbot, D.Liepmann, A.P.Pisano, "Micro-fabricated Polysilicon Microneedles for Minimally Invasive Biomedical Devices Biomedical Microdevices" Vol 2, (2000), pp. 295-303.

[3] S. Henry, D.V. McAllister, M. G. Allen, M. R. Prausnitz, "Microfabricated microneedles: A novel approach to transdermal drug delivery", Journal of Pharmaceutical Sciences, Vol. 87, No. 8, August, (1998), pp. $922-925$.

[4] J.Gere, S. Timoshenko, "Mechanics of Materials", fourth ed, May 1997

[5] Shankar Chandrasekhar, A Bruno Frazier, "Mechanical Characterization of Surface Micromachined Hollow Metallic Microneedles", Microtechnologies in Medicine \& Biology 2nd Annual International IEEE-EMB Special Topic Conference, 2-4 May 2002, pp. $94-98$

[6] PN Brett, T J Parker, A J Harrison, T A Thomas and A Carr, "Simulation of Resistance Forces Acting on the Surgical Needles" $2^{\text {nd }}$ International Workshop on Mechatronics in Medicine and Surgery, Bristol, 1997

[7] Marc Madou, "Fundamentals of Microfabrication", CRC Press, 1997 